\title{
Structural and optical properties of silicon-germanium alloy nanoparticles
}

\author{
Chung-Wei Lin, Shih-Yen Lin, and Si-Chen Lee ${ }^{\mathrm{a})}$ \\ Department of Electrical Engineering and Graduate Institute of Electronic Engineering, National Taiwan \\ University, Taipei, Taiwan, Republic of China \\ Chih-Ta Chia \\ Department of Physics, National Normal Taiwan University, Taipei, Taiwan, Republic of China
}

(Received 18 June 2001; accepted for publication 14 November 2001)

\begin{abstract}
Silicon-germanium alloy nanoparticles with spherical shape have been prepared by the thermal evaporation method. The shape and structure of these dots have been studied. Transmission electron microscopy images show that the SiGe nanoparticles grown at 100 Torr may be composed of two half-moon shaped sections. Furthermore from the transmission electron diffraction patterns and Raman spectra, a change of structure from amorphous to crystalline at the 0.4 Torr growth pressure was observed. It is also found that if the growth pressure increases, the Ge composition of SiGe dots will decrease. A model is proposed to explain this phenomenon. (c) 2002 American Institute of Physics. [DOI: 10.1063/1.1433185]
\end{abstract}

\section{INTRODUCTION}

In recent years, nanoparticles have attracted much attention because of their physical and optical properties and potential applications in optoelectronic and quantum devices, ${ }^{1-5}$ such as a single electron transistor. ${ }^{6-8}$ There are many methods to prepare nanoparticles. The techniques include chemical precipitation, ${ }^{9}$ thermal evaporation, ${ }^{10-12}$ chemical vapor deposition, ${ }^{13,14}$ laser ablation, ${ }^{15}$ sputtering, ${ }^{16-18}$ and molecular beam epitaxy, ${ }^{19}$ etc. The thermal evaporation method was chosen to synthesize SiGe quantum dots ${ }^{20}$ because of its simplicity. It can control the dot size by adjusting the gas pressure in the chamber and composition by altering the boat current without any other complicated processes.

\section{EXPERIMENTS}

The substrate was 7059 glass and was cleaned in the following steps: dipped in $\mathrm{H}_{2} \mathrm{SO}_{4}: \mathrm{H}_{2} \mathrm{O}_{2}$ (3:1) for $30 \mathrm{~min}$, rinsed in de-ionized water for $10 \mathrm{~min}$, then blown dry with $\mathrm{N}_{2}$ gas in order to wash away organic matters. The $\mathrm{Si}$ or $\mathrm{Ge}$ powders were placed in the respective tantalum $(\mathrm{Ta})$ boats and the temperature was controlled by adjusting the electric current through the boat which provided different evaporation rates. The distance between the boats and the substrate was $13 \mathrm{~cm}$. The air in the chamber was pumped out to about $10^{-4}$ Torr and the chamber was purged with Ar gas three times to get rid of residual water vapor and oxygen. Then the chamber was filled with Ar to the desired pressure and the substrate was cooled down by the cold trap filled with liquid nitrogen. After the filament temperature was raised to the desired one, the shutter between the source and substrate was opened and the nanoparticles were deposited on the substrate. Here, the boat temperatures were set at 1500 and

a) Author to whom correspondence should be addressed; electronic mail: sclee@cc.ee.ntu.edu.tw
$1400{ }^{\circ} \mathrm{C}$ for $\mathrm{Si}$ and Ge boats, respectively. Finally, the specimens were warmed to room temperature and taken out of the chamber.

\section{RESULTS AND DISCUSSION}

The transmission electron microscopy (TEM) images of SiGe nanoparticles grown at 0.2, 0.4, 1 and 100 Torr are shown in Fig. 1. In Fig. 1(a), it can be seen that there is no dot-like structure existing, it looks like the coarse film. But in Figs. 1(b)-1(d), the SiGe particles appear and their size becomes larger, generally with larger growth pressure. The sizes of $\mathrm{SiGe}$ particles are $11.5 \pm 0.7,18.9 \pm 3.1$, and 56.8 $\pm 16.3 \mathrm{~nm}$ for the samples shown in Figs. 1(b), 1(c), and $1(\mathrm{~d})$, respectively. Besides, the shape of particles is very clear and almost no coarse film exists between them while the growth pressure reaches 1 Torr. Something interesting is observed in Fig. 1(d). If particles are partly overlapped, the gibbous shape will be seen and it could often be seen in different TEM images. But in the central region of Fig. 1(d), a nanoparticle composed of two half-moon shaped sections is observed. One section looks darker and the other looks brighter. They must consist of two different materials or the same material with different orientations. If the growth pressure of SiGe nanoparticles is below 1 Torr, it disappears. The reason may be attributed to the large growth pressure. Before the Si and Ge particles collide with each other, they have collided with the same species for many times, so the particle sizes are already large when they collide with each other. When this happens, the particle with two half-moon shaped sections may be produced. In addition, two Si or Ge particles with different orientation may also be produced this way.

Figures 2(a) and 2(b) display the transmission electron diffraction (TED) patterns of SiGe nanoparticles grown at 0.2 and 0.4 Torr, respectively. In Fig. 2(a), no diffraction rings are observed, so only amorphous $\mathrm{SiGe}$ is produced. This is consistent with Fig. 1(a), that only a coarse film is formed, no crystalline nanoarticles could be seen. Figure 


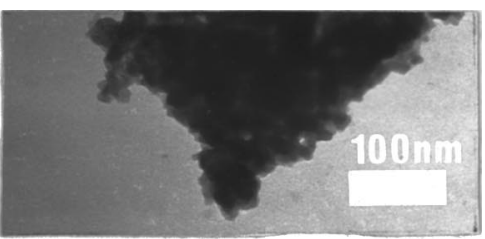

(a)

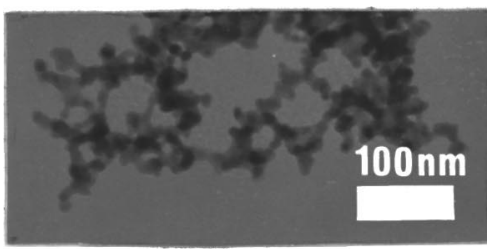

(b)

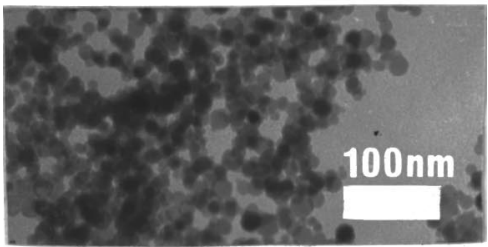

(C)

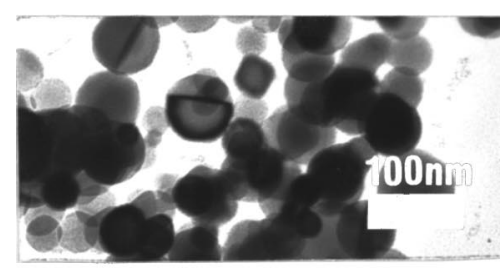

(d)

FIG. 1. The TEM images of SiGe nanoparticles grown at: (a) 0.2, (b) 0.4, (c) 1 , and (d) 100 Torr.

2(b) shows the (111) and (220) diffraction ring pattern indicating the formation of crystalline structure. A critical size seems to exist for the SiGe nanoparticle below which no crystalline particle can be produced. This is probably caused by the expansion of the lattice constant. If the particle size is too small, the diamond structure may become unstable because of the large fraction of surface dangling bonds. They may expand and collapse to form amorphous structure and land on the substrate to form coarse film. From the diffraction pattern, it is also clear that $\mathrm{Si}$ and $\mathrm{Ge}$ are mixed together completely, no pure $\mathrm{Si}$ or Ge particles are formed. By comparing the (220) ring in Fig. 2(b) with the polycrystalline $\mathrm{Si}$, the lattice constant of SiGe nanoparticles is calculated to be $5.54 \AA$. The value is just between the lattice constant of diamond structure $\mathrm{Si}(5.43 \AA)$ and that of diamond structure Ge $(5.66 \AA)$. This proves that the particles are really SiGe alloy. The TED patterns of SiGe particles grown at 1 and 100 Torr are shown in Figs. 3(a) and 3(b), respectively, the rings are clearer than those shown in Fig. 2, and many bright spots are observed. This indicates that the particle structure becomes better and is spatially oriented. From the radius of the diffraction ring, the lattice constant of the SiGe nanoparticle can be extracted. According to Vegard's law, ${ }^{21}$ the relationship between the lattice constant $a$ and $\mathrm{Ge}$ content of $\mathrm{Si}_{1-x} \mathrm{Ge}_{x}$ at $300 \mathrm{~K}$ is

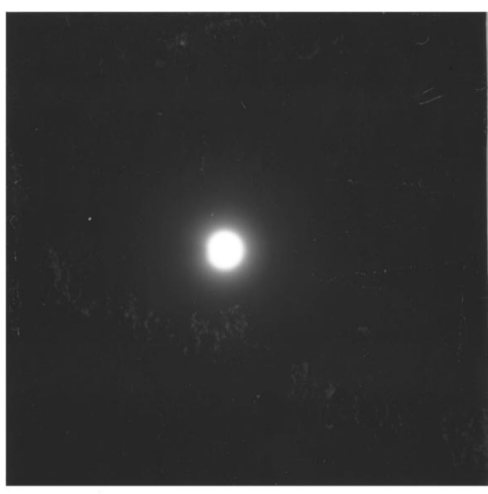

(a)

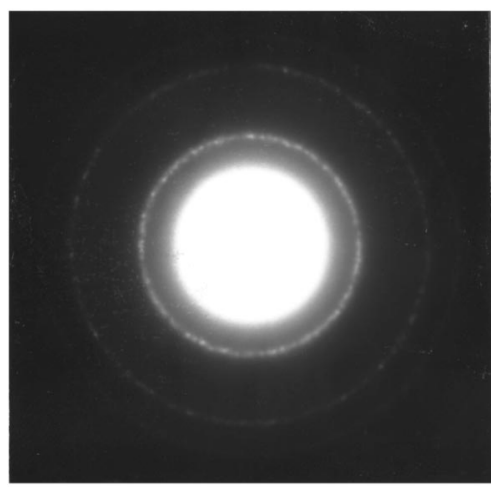

(b)

FIG. 2. The TED patterns of SiGe particles grown at: (a) 0.2 and (b) 0.4 Torr.

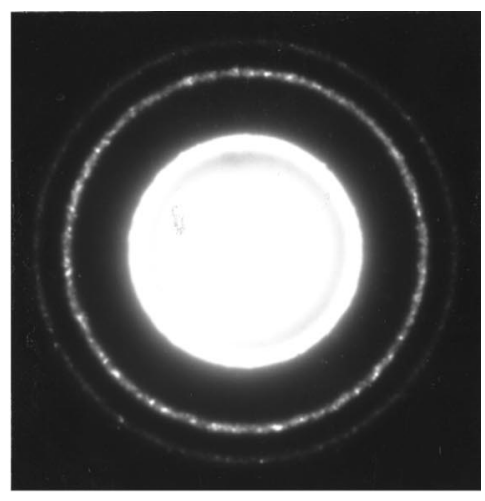

(a)

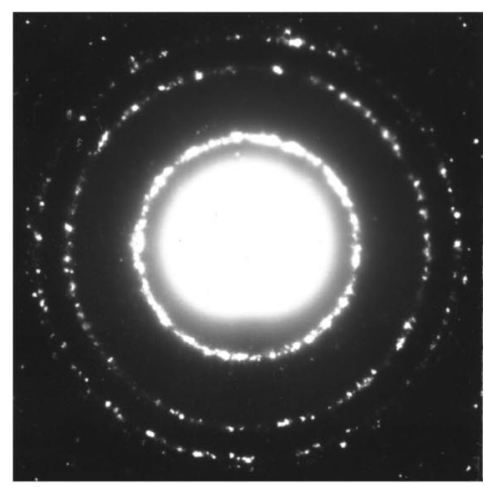

(b)

FIG. 3. The TED patterns of SiGe particles grown at: (a) 1 and (b) 100 Torr. 


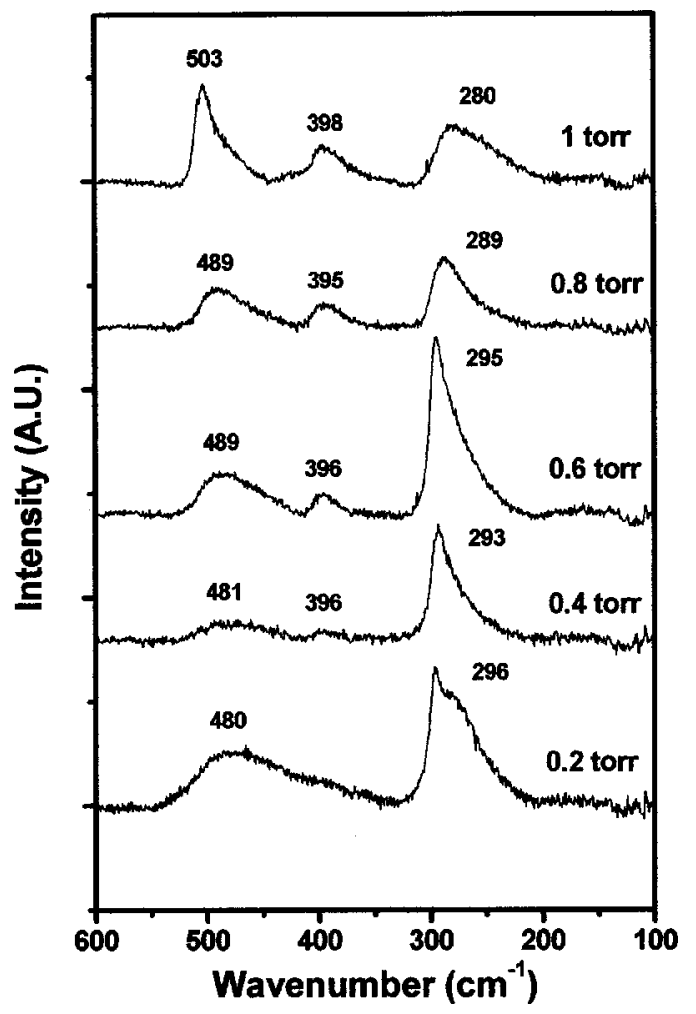

FIG. 4. The Raman spectra of SiGe particles.

$a=5.431+0.227 x$.

The Ge composition of SiGe nanoparticles grown at 0.4 and 100 Torr are 0.47 and 0.31 , respectively, and only the (220) ring is considered because it is clearer and larger, which can minimize the calculation error. It is clear that Ge composition becomes smaller when the growth pressure becomes larger, therefore, by controlling the growth pressure, the $\mathrm{Ge}$ composition of the SiGe nanoparticles can be adjusted.

Shown in Fig. 4 are the Raman spectra of SiGe dots grown at different pressure from 0.2 to 1 Torr. The Raman spectra were measured using an Ar laser with $20 \mathrm{~mW}$ power. The nanoparticles are grown on 7059 glass where the peak is located at $480 \mathrm{~cm}^{-1}$. The peak is broad and mixed with the peak of SiGe nanoparticles. However, when the nanoparticle layer becomes thicker, the effect of the glass substrate can be ignored. Three peaks are observed, which are located at about 500,400 , and $300 \mathrm{~cm}^{-1}$, corresponding to the vibration of $\mathrm{Si}-\mathrm{Si}, \mathrm{Si}-\mathrm{Ge}$, and $\mathrm{Ge}-\mathrm{Ge}$ phonons, respectively, so the alloy particles are really formed instead of a mixture of pure $\mathrm{Si}$ or $\mathrm{Ge}$ particles. It is clear that when the growth pressure is 0.2 Torr, SiGe crystalline particles are not produced. Only amorphous $\mathrm{Si}$ and $\mathrm{Ge}$ exist. This matches the TEM result in Fig. 1(a) very well. While the growth pressure reaches 0.4 Torr, $\mathrm{SiGe}$ particles appear. It can be seen that $\mathrm{Si}$ and Ge are mixed completely, so the peak at $400 \mathrm{~cm}^{-1}$ appears. It is also found that three peak positions change when the growth pressure is changed. Because the variation of the $\mathrm{Si}-\mathrm{Si}$ peak position is larger and easier to compare, the $\mathrm{Ge}$ composition is estimated from it. Alonso and Winer ${ }^{22}$ have studied the relationship between the peak position and $\mathrm{Ge}$ content in the $\mathrm{Si}_{1-x} \mathrm{Ge}_{x}$ bulk which could be written as

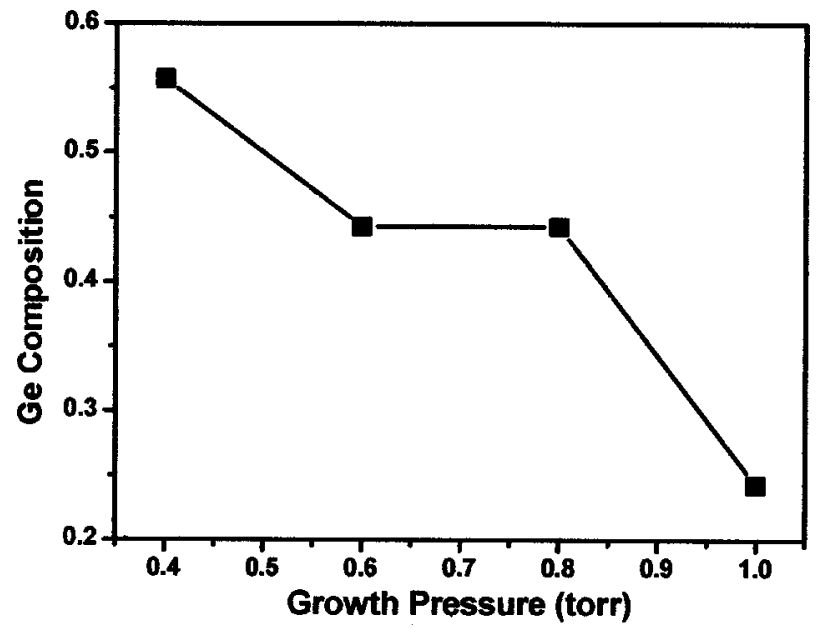

FIG. 5. The Ge composition as a function of the growth pressure of SiGe particles.

$$
y=520-70 x
$$

where $y$ is the peak position of the $\mathrm{Si}-\mathrm{Si}$ phonon and $x$ is the fraction of $\mathrm{Ge}$ in the bulk. Based on this equation, the $\mathrm{Ge}$ fraction in the particles could be estimated. Figure 5 displays the relationship between the growth pressure and the Ge composition. It can be seen that if the growth pressure increases, the Ge composition decreases. But the values do not match the values obtained from TED results. This may be caused by the different spot size of the electron beam and laser. The reason why the composition changes may be attributed to the probability of collision. Two boats are located at a distance about $1 \mathrm{~cm}$, so before two kinds of atoms collide with each other they have already formed grains of their own kind. Since the Si boat temperature is $1500{ }^{\circ} \mathrm{C}$ and about $100{ }^{\circ} \mathrm{C}$ higher than the temperature of the Si melting point, and the $\mathrm{Ge}$ boat temperature is $1400{ }^{\circ} \mathrm{C}$ and about $400{ }^{\circ} \mathrm{C}$ higher than the temperature of the Ge melting point, the $\mathrm{Ge}$ grain size should be larger. When the large Ge grain collides with the small Si grain, it is obvious that the $\mathrm{Si}$ grain will be sprung easily, so the Ge composition of the dot is larger. But when the growth pressure becomes larger, the Si grain size

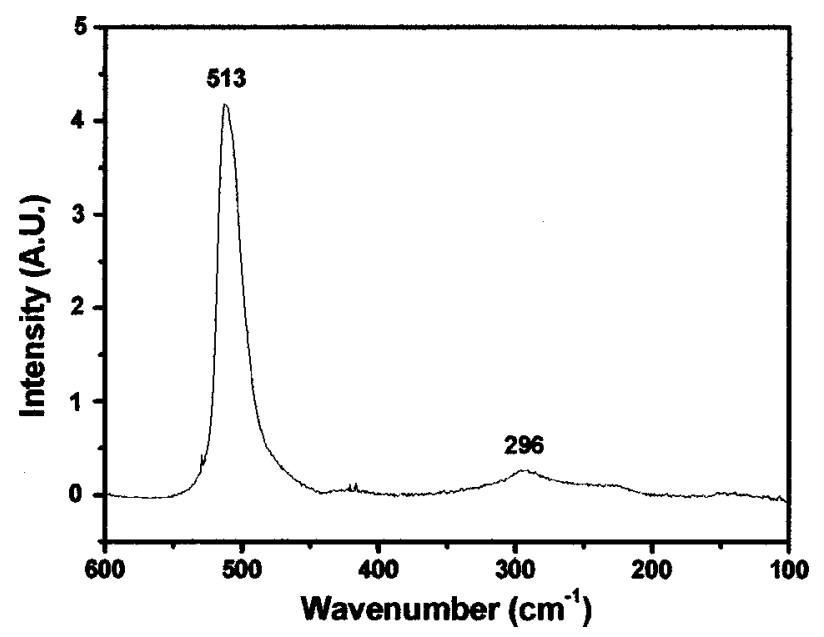

FIG. 6. The Raman spectrum of SiGe particles grown at 100 Torr. 
may grow quickly. Because the melting point of $\mathrm{Si}$ is higher than that of Ge, the Si nucleation is quicker and the Ar gas may speed up the nucleation. Therefore, the Si grain size will become larger than the Ge grain size and the Si composition will increase. In order to make sure this assumption is reasonable, the Raman spectrum of SiGe particles grown at 100 Torr is measured and shown in Fig. 6. It is clear that the $\mathrm{Si}-\mathrm{Si}$ peak at $513 \mathrm{~cm}^{-1}$ is very strong and the Ge peak at $296 \mathrm{~cm}^{-1}$ is barely seen, suggesting that the Ge composition is very small. The Si peak is at $513 \mathrm{~cm}^{-1}$, not at $521 \mathrm{~cm}^{-1}$, indicating the crystallinity of the SiGe nanoparticle is not perfect. The influence of Ge atoms although small cannot be ignored. It is concluded that the Ge composition of $\mathrm{SiGe}$ nanoparticles will decrease if the growth pressure gets larger.

\section{CONCLUSIONS}

The crystalline SiGe nanoparticles will be produced when the growth pressure is higher than 0.4 Torr. When the growth pressure reaches 100 Torr, several SiGe nanoparticles exhibiting two half-moon shaped sections appear. If the growth pressure becomes larger, the Ge composition of SiGe nanoparticles will become smaller. The critical Ge composition of $\mathrm{SiGe}$ nanoparticles growth at 0.4 Torr is about 0.5 .

\section{ACKNOWLEDGMENT}

This work is supported by the National Science Council of the Republic of China under Contract No. NSC90-2215E002-010.
${ }^{1}$ Y. S. Tang, C. M. Sotomayor Torres, W. X. Ni, and G. V. Hansson, Superlattices Microstruct. 20, 505 (1996).

${ }^{2}$ L. J. Guo and S. Y. Chou, Electron. Lett. 34, 1030 (1998).

${ }^{3}$ H. Tamura, M. Ruckschloss, T. Wirschem, and S. Veprek, Thin Solid Films 255, 92 (1998).

${ }^{4}$ H. Morisaki, H. Hashimoto, F. W. Ping, H. Nozawa, and H. Ono, J. Appl. Phys. 74, 2977 (1993).

${ }^{5}$ S. Gardelis, J. S. Rimmer, P. Dawson, and B. Hamilton, Appl. Phys. Lett. 59, 2118 (1991).

${ }^{6}$ H. Ishikuro and T. Hiramoto, Appl. Phys. Lett. 71, 3691 (1997).

${ }^{7}$ R. Augke, W. Eberhardt, C. Single, F. E. Prins, D. A. Wharam, and D. P. Kem, Appl. Phys. Lett. 76, 2065 (2000).

${ }^{8}$ A. Nakajima, T. Futatsugi, K. Kosemura, and T. Fukano, J. Vac. Sci. Technol. B 17, 2163 (1999).

${ }^{9}$ Z. X. Tang, C. M. Sorenson, K. J. Klabunde, and G. C. Hadjipanayis, J. Colloid Interface Sci. 146, 38 (1991).

${ }^{10}$ Y. Kawamura, M. Takagi, and M. Akai, Mater. Sci. Eng. 98, 449 (1988).

${ }^{11}$ C. G. Granqvist and R. A. Buhrman, J. Appl. Phys. 47, 2200 (1976).

${ }^{12}$ R. W. Siegal, Mater. Sci. Eng., A 168, 189 (1993).

${ }^{13}$ Y. He, H. Liu, N. Yu, and X. M. Yu, Nanostruct. Mater. 7, 769 (1996).

${ }^{14}$ Y. He, C. Yin, G. Cheng, L. Wang, and X. Liu, J. Appl. Phys. 75, 797 (1994).

${ }^{15}$ N. L. Mandich, V. E. Bondybey, and W. D. Reents, J. Chem. Phys. 86, 4245 (1987).

${ }^{16}$ H. Hahn and R. S. Averback, J. Appl. Phys. 67, 1113 (1990).

${ }^{17}$ G. M. Chow, C. L. Chien, and A. S. Edelstein, J. Mater. Sci. 6, 8 (1991).

${ }^{18}$ V. Hass and R. Birringer, Nanostruct. Mater. 1, 491 (1992).

${ }^{19}$ E. Gaffet and M. Harmelin, J. Less-Common Met. 157, 201 (1990).

${ }^{20}$ Y.-C. Lio, S.-Y. Lin, S.-C. Lee, and C.-T. Chia, Appl. Phys. Lett. 77, 4328 (2000).

${ }^{21}$ P. Becker, P. Seyfried, and H. Siegert, Z. Phys. B: Condens. Matter 48, 17 (1982).

${ }^{22}$ M. I. Alonso and K. Winer, Phys. Rev. B 39, 10056 (1989). 